\title{
A PROOF OF THE POPOV CONJECTURE FOR TORI
}

\author{
DAVID L. WEHLAU
}

(Communicated by Jonathan M. Rosenberg)

\begin{abstract}
We prove a lemma which reduces much of the invariant theory of torus representations to the theory of faithful stable torus representations (Lemma 2). Using this reduction we obtain a structure theorem (Theorem 1) for equidimensional representations of tori. This theorem shows that the weights of an equidimensional torus representation are arranged in a very special manner within the lattice of characters. Understanding this arrangement allows us to prove that equidimensional representations of tori must be cofree (the Popov conjecture for tori).
\end{abstract}

\section{INTRODUCTION}

Let $G$ be a complex reductive algebraic group and $V$ a $G$-module. Denote by $\mathbb{C}[V]$ the ring of polynomial functions on $V$. If $\left\{x_{1}, \ldots, x_{n}\right\}$ is a basis for $V^{*}$, the dual of $V$, then $\mathbb{C}[V]$ is just the polynomial ring $\mathbb{C}\left[x_{1}, \ldots, x_{n}\right]$. The action of $G$ on $V$ induces an action of $G$ on $\mathbb{C}[V]$ given by $(g \cdot f)(v)=$ $f\left(g^{-1} v\right)$ for $f \in \mathbb{C}[V], g \in G$, and $v \in V$. The elements of $\mathbb{C}[V]$ which are fixed by $G$ form a subring, called the ring of $G$-invariants and denoted $\mathbb{C}[V]^{G}$. The ring of invariants of a reductive group is finitely generated and so is the coordinate ring of an affine variety. This variety is called the quotient of $V$ by $G$ and denoted by $V / / G$. Dual to the inclusion of rings $\mathbb{C}[V]^{G} \hookrightarrow \mathbb{C}[V]$ we have a morphism of affine varieties, the quotient morphism, $\pi_{V, G}: V \rightarrow V / / G$.

We are interested in three properties of representations: coregularity, equidimensionality, and cofreeness. If $\mathbb{C}[V]^{G}$ is a regular ring then $(V, G)$ is said to be coregular. A representation $(V, G)$ is called equidimensional if all the fibres of $\pi_{V, G}$ have the same dimension. If $\mathbb{C}[V]$ is a free $\mathbb{C}[V]^{G}$-module then $(V, G)$ is cofree. A representation is cofree if and only if it is both coregular and equidimensional [11].

The Popov conjecture is that if $G$ is connected then every equidimensional representation of $G$ is also cofree. We prove that the conjecture is valid if $G$ is a torus and give a complete description of the torus representations to which it applies in terms of their weights.

Received by the editors April 12, 1990 and, in revised form, October 7, 1990.

1991 Mathematics Subject Classification. Primary 22E46; Secondary 20 G05.

Research supported in part by NSERC Grant OGP0041784. 


\section{PRELIMINARIES}

We begin with some standard results. Proofs for these results may be found in [5]. Let $G$ be a complex reductive algebraic group. We will use $(V, G)$ to denote a complex representation of $G$ with representation space $V$.

The quotient morphism, $\pi_{V, G}: V \rightarrow V / / G$, gives a bijection between the points of $V / / G$ and the closed $G$-orbits in $V$. Notice that if $\left\{f_{1}, \ldots, f_{d}\right\}$ is a generating set for $\mathbb{C}[V]^{G}$, then $\pi_{V, G}$ can be realized as $\pi_{V, G}=\left(f_{1}, \ldots, f_{d}\right)$ : $V \rightarrow \mathbb{C}^{d} \supset V / / G$ where $V / / G \cong$ Image of $\pi_{V, G}$.

An important property of the invariants is that they suffice to separate closed $G$-stable subsets of $V$. That is, if $V_{1}$ and $V_{2}$ are disjoint closed nonempty $G$-stable subsets of $V$, then there exists $v_{1} \in V_{1}$ and $v_{2} \in V_{2}$ with $\pi_{V, G}\left(v_{1}\right) \neq$ $\pi_{V, G}\left(v_{2}\right)$.

The $\mathbb{N}$ grading on $\mathbb{C}[V]$ by total polynomial degree is preserved by the action of $G$. Hence $\mathbb{C}[V]^{G}$ is also $\mathbb{N}$-graded. From this it follows that $(V, G)$ is coregular if and only if $\mathbb{C}[V]^{G}$ is a polynomial ring. Hence $(V, G)$ is coregular if and only if $V / / G$ is affine space $\mathbb{C}^{d}$.

Next we derive an important characterization of equidimensionality. The quotient, $V / / G$, always contains a dense open subset $A$ such that $\operatorname{dim} \pi_{V, G}^{-1}(\xi)=$ $\operatorname{dim} V-\operatorname{dim} V / / G \forall \xi \in A$. Moreover every component of every fibre of $\pi_{V, G}$ has dimension at least $\operatorname{dim} V-\operatorname{dim} V / / G$. Conversely, it is well known that $\operatorname{dim} V^{\circ} \geq \operatorname{dim} \pi_{V, G}^{-1}(\xi) \forall \xi \in V / / G$ where $V^{\circ}:=\pi_{V, G}^{-1}\left(\pi_{V, G}(0)\right)$, is the null cone or null fibre. Therefore

$$
\begin{aligned}
(V, G) \text { is equidimensional } & \Longleftrightarrow \operatorname{dim} V^{\circ}=\operatorname{dim} V-\operatorname{dim} V / / G \\
& \Longleftrightarrow \operatorname{dim} V^{\circ} \leq \operatorname{dim} V-\operatorname{dim} V / / G .
\end{aligned}
$$

In 1976, V. Kac, V. Popov, and E. Vinberg classified the coregular irreducible representations of connected simple groups [4]. In [8], Popov observed that these representations are precisely the cofree irreducible representations of connected simple groups. This led Popov to make the conjecture that every equidimensional representation of a connected semisimple group is also cofree.

There are many equidimensional representations of nonconnected semisimple groups (for example finite groups) which are not coregular. However the necessity of semisimpleness as a hypothesis in the conjecture is not so clear and in 1980, V. Kac extended the Popov conjecture by conjecturing that for any connected complex algebraic group whatsoever, every equidimensional representation is cofree [3]. We know of no counterexamples to even this extended version of the Popov conjecture.

G. Schwarz listed the coregular representations of connected simple groups in 1978 [9]. That same year, Schwarz also listed the cofree representations of connected simple groups [10]. These lists show that there are many reducible coregular representations of connected simple groups which are not equidimensional. In 1979, O. Adamovich and E. Golovina also classified the coregular representations of connected simple groups [2]. Using methods from his two classifications, Schwarz verified the Popov conjecture for connected simple groups (unpublished). Independently, Adamovich also verified the conjecture for these groups [1]. 
P. Littelmann listed, in 1987 (up to the equivalence relation known as castling), for connected semisimple groups, the cofree, coregular, and equidimensional representations which are irreducible [6]. These lists show that the Popov conjecture is true for irreducible representations of connected semisimple groups.

The current author has listed the equidimensional representations of a large class of the connected 2-simple groups [13]. Again this list verifies the conjecture for these groups.

The proofs that the conjecture is valid for these special cases all involve explicitly listing all the relevant representations which are either coregular or equidimensional. Once these classifications have been done it is seen that all the equidimensional representations of the given type are also coregular. In contrast to these other proofs we will give a direct proof that the conjecture is valid for torus representations.

We still require one more definition and one important result. A representation $(V, G)$ is stable if the union of the closed $G$-orbits in $V$ contains an open dense subset of $V$.

One of the most important methods for determining whether an orbit is closed is the

Hilbert-Mumford criterion. If $y \in \overline{G \cdot x}$, then there exits a one parameter subgroup $\lambda: \mathbb{C}^{*} \rightarrow G$ and an element $g \in G$ such that

$$
\lim _{t \rightarrow 0} \lambda(t) \cdot x=g \cdot y \quad \text { (in the classical topology). }
$$

\section{TORUS REPRESENTATIONS}

Now we take $G=T \cong\left(\mathbb{C}^{*}\right)^{r}$ a torus of dimension $r$. Let $X^{*}(T) \cong \mathbb{Z}^{r}$, be the lattice of characters of $T$. Given $(V, T)$, a representation of $T$, we may choose a basis $\left\{v_{1}, \ldots, v_{n}\right\}$ of $V$ consisting of weight vectors. Let $\nu_{i}$ be the weight of $v_{i}$. Let $\left\{x_{1}, \ldots, x_{n}\right\}$ be the corresponding dual basis of $V^{*}$. Then each $x_{i}$ is a semiinvariant of weight $\mu_{i}:=-\nu_{i}$. Moreover, there are minimal generating sets for $\mathbb{C}[V]^{T}$ of the form $\left\{m_{1}, \ldots, m_{s}\right\}$ where the $m_{j}$ are (nonconstant) monomials in the $x_{i}$. It is the existence of these minimal generating sets consisting of monomials that makes the invariant theory of torus representations accessible.

Whenever we have a torus representation $(V, T)$, we will use $v_{i}$ to denote elements of such a basis of $T$-weight vectors, $x_{i}$ to denote the corresponding dual semiinvariants, and $\mu_{i}=-\nu_{i}$ to denote the weight of $x_{i}$. Furthermore, whenever we choose generating sets $\left\{m_{1}, \ldots, m_{s}\right\}$ for $\mathbb{C}[V]^{T}$, we will suppose that the $m_{j}$ are (nonconstant) monomials in the $x_{i}$.

A proof of the following lemma may be found in $[12, \S 2.5]$.

Lemma 1. If $T$ is a torus and $X^{*}(T)=M_{1} \oplus M_{2}$, then $T=T_{1} \times T_{2}$ where $T_{1}=\bigcap_{\chi \in M_{2}} \operatorname{ker} \chi, T_{2}=\bigcap_{\chi \in M_{1}} \operatorname{ker} \chi$, and $X^{*}\left(T_{i}\right)=M_{i}$ for $i=1,2$.

Now we prove a lemma which allows us to reduce to stable faithful representations.

Lemma 2. Let $(V, T)$ be a representation of a torus. Let $\left\{v_{1}, \ldots, v_{n}\right\}$ be $a$ basis for $V$ and $\left\{m_{1}, \ldots, m_{s}\right\}$ a generating set for $\mathbb{C}[V]^{T}$. Suppose that 
$\left\{v_{1}, \ldots, v_{k}\right\}=\left\{v_{i} \mid \exists j\right.$ with $x_{i}$ dividing $\left.m_{j}\right\}$, define $V^{\prime}:=\operatorname{span}_{\mathbb{C}}\left\{v_{1}, \ldots, v_{k}\right\}$ and $N:=\operatorname{span}_{\mathbb{C}}\left\{v_{k+1}, \ldots, v_{n}\right\}$. Set $H:=\operatorname{ker}\left(T \rightarrow G L\left(V^{\prime}\right)\right)$ and $T^{\prime}:=T / H$. Then

(1) $\mathbb{C}\left[V^{\prime}\right]^{T^{\prime}}=\mathbb{C}[V]^{T}$.

(2) $\mathbb{C}[N]^{T}=\mathbb{C}$.

(3) $\left(V^{\prime}, T^{\prime}\right)$ is a faithful stable torus representation.

(4) $\pi_{V, T}\left(v^{\prime}+n\right)=\pi_{V^{\prime}, T^{\prime}}\left(v^{\prime}\right)$ for all $v^{\prime} \in V^{\prime}, n \in N$.

(5) $(V, T)$ is stable $\Longleftrightarrow V=V^{\prime}$.

(6) $(V, T)$ is cofree (respectively coregular, equidimensional) $\Longleftrightarrow\left(V^{\prime}, T^{\prime}\right)$ is cofree (respectively coregular, equidimensional).

Proof. Both (1) and (2) are clear.

For (3), $\left(V^{\prime}, T^{\prime}\right)$ is faithful by definition of $T^{\prime}$. To see that $\left(V^{\prime}, T^{\prime}\right)$ is stable let $B:=\left\{v^{\prime}=\left(v_{1}^{\prime}, \ldots, v_{k}^{\prime}\right) \in V^{\prime} \mid v_{i}^{\prime} \neq 0\right.$ for $\left.i=1, \ldots, k\right\}$. Then $B$ is an open subset of $V^{\prime}$ and we will now show that all $v^{\prime} \in B$ lie on closed $T^{\prime}$-orbits. Let $\lambda: \mathbb{C}^{*} \rightarrow T$ be a nonconstant one parameter subgroup. Suppose $x_{i}$ has weight $\alpha_{i} \in X^{*}\left(\lambda\left(\mathbb{C}^{*}\right)\right) \cong \mathbb{Z}$. Since $\lambda$ is not constant and $\left(V^{\prime}, T^{\prime}\right)$ is faithful, there exists $i_{0}$ such that $\alpha_{i_{0}} \neq 0$. By hypothesis, there exists $j_{0}$ such that $x_{i_{0}}$ divides $m_{j_{0}}$. Since $m_{j_{0}}$ has weight 0 there exists $i_{1}$, such that $x_{i_{1}}$ divides $m_{j_{0}}, \alpha_{i_{1}} \neq 0$, and $\alpha_{i_{0}}$ and $\alpha_{i_{1}}$ have different sign. But then $v_{i_{0}} \neq 0$ and $v_{i_{1}} \neq 0$ implies that

$$
\lim _{t \rightarrow 0} \lambda(t) \cdot v^{\prime}=\lim _{t \rightarrow 0}\left(t^{\alpha_{1}} v_{1}^{\prime}, \ldots, t^{\alpha_{i_{0}}} v_{i_{0}}^{\prime}, \ldots, t^{\alpha_{i_{1}}} v_{i_{1}}^{\prime}, \ldots, t^{\alpha_{n}} v_{n}^{\prime}\right)
$$

does not exist. Hence by the Hilbert-Mumford criterion, $T \cdot v^{\prime}=T^{\prime} \cdot v^{\prime}$ is closed.

Since we have $\pi_{V, T}=\left(m_{1}, \ldots, m_{s}\right): V \rightarrow V / / T,(4)$ is clear.

To prove (5) consider $v^{\prime} \in B$. By the proof of $(3), T \cdot v^{\prime}=T^{\prime} \cdot v^{\prime}$ is a closed orbit in $V^{\prime}$, hence $T \cdot v^{\prime}$ is also closed in $V$. Now the invariants separate closed orbits and thus $T \cdot\left(v^{\prime}+n\right)$ cannot be closed if $n \neq 0$. Therefore

$$
\left\{v^{\prime}+n \mid v^{\prime} \in B \text { and } 0 \neq n \in N\right\}
$$

consists only of nonclosed orbits.

To prove (6) we apply (1) to see that $(V, T)$ is coregular iff if $\left(V^{\prime}, T^{\prime}\right)$ is coregular. By the proof of $(5), \pi_{V, T}^{-1}\left(\pi_{V, T}\left(v^{\prime}, n\right)\right)=\pi_{V^{\prime}, T^{\prime}}^{-1}\left(\pi_{V^{\prime}, T^{\prime}}\left(v^{\prime}\right)\right) \times N$ and thus $(V, T)$ is equidimensional if and only if $\left(V^{\prime}, T^{\prime}\right)$ is equidimensional.

Remark 1.

$$
\begin{aligned}
(V, T) \text { is faithful } & \Longleftrightarrow \operatorname{span}_{\mathbb{Z}}\left\{\nu_{1}, \ldots, \nu_{n}\right\}=X^{*}(T) \\
& \Longleftrightarrow \operatorname{span}_{\mathbb{Z}}\left\{\mu_{1}, \ldots, \mu_{n}\right\}=X^{*}(T) .
\end{aligned}
$$

Remark 2. We may write $V^{\prime}$ as a disjoint union of closed $T^{\prime}$-stable sets:

$$
V^{\prime}=\bigsqcup_{v^{\prime} \in B} T^{\prime} \cdot v^{\prime} \bigsqcup\left(V^{\prime} \backslash B\right) .
$$

Since $\pi_{V^{\prime}, T^{\prime}}$ separates closed $T^{\prime}$-stable subsets, this decomposition shows that $\pi_{V^{\prime}, T^{\prime}}^{-1}\left(\pi_{V^{\prime}, T^{\prime}}\left(v^{\prime}\right)\right)=T^{\prime} \cdot v^{\prime} \forall v^{\prime} \in B$. Now since $\left(V^{\prime}, T^{\prime}\right)$ is faithful we see that 
$\operatorname{dim} T^{\prime} \cdot v^{\prime}=\operatorname{dim} T^{\prime} \forall v^{\prime} \in B$. Thus, almost all fibres of $\pi_{V^{\prime}}, T^{\prime}$ have dimension equal to $\operatorname{dim} T^{\prime}$. Therefore $\operatorname{dim} V^{\prime} / / T^{\prime}=\operatorname{dim} V^{\prime}-\operatorname{dim} T^{\prime}$.

\section{EQUIDIMENSIONAL REPRESENTATIONS}

Theorem 1 (Structure theorem for equidimensional torus representations). If $(V, T)$ is a faithful, stable, equidimensional torus representation with $\operatorname{dim} V=$ $n$ and $\operatorname{dim} T=r$, then $(V, T) \cong\left(V_{1}, T_{1}\right) \oplus \cdots \oplus\left(V_{n-r}, T_{n-r}\right)$ where $V=$ $V_{1} \oplus \cdots \oplus V_{n-r}, \quad T=T_{1} \times \cdots \times T_{n-r}$, and $\left(V_{j}, T_{j}\right)$ is a faithful, stable, cofree torus representation with $\operatorname{dim} V_{j} / / T_{j}=1$ for $j=1, \ldots, n-r$. Moreover the weights of each of the $V_{j}$ are arranged within $X^{*}\left(T_{j}\right) \cong \mathbb{Z}^{\left(\operatorname{dim} V_{j}-1\right)}$ in such a manner that they are linearly dependent over $\mathbb{N}$, but any $\operatorname{dim} V_{j}-1$ of them form a basis for $X^{*}\left(T_{j}\right) \otimes \mathbb{Q}$.

Proof. Let $\left\{x_{1}, \ldots, x_{n}\right\}$ be a basis of $V^{*}$ and let $\left\{m_{1}, \ldots, m_{d}\right\}$ be a minimal generating set for $\mathbb{C}[V]^{T}$. By Remark $2, \operatorname{dim} V / / T=\operatorname{dim} V-\operatorname{dim} T=n-r$.

We say that the set $\left\{x_{i_{1}}, \ldots, x_{i_{k}}\right\}$ satisfies property $\star$ if for all $j$ with $1 \leq j \leq d$ there exists a $p$ with $1 \leq p \leq k$ such that $x_{i_{p}}$ divides $m_{j}$. Note that

$$
V^{0}=\bigcup_{k} \bigcup_{\substack{\left\{x_{i_{1}}, \ldots, x_{i_{k}}\right\} \\ \text { satisfies } *}} H\left(i_{1}, \ldots, i_{k}\right)
$$

where $H\left(i_{1}, \ldots, i_{k}\right)$ is the $n-k$ dimensional $T$-stable subspace of $V$ defined by $H\left(i_{1}, \ldots, i_{k}\right):=\left\{\left(v_{1}, \ldots, v_{n}\right) \in V \mid v_{i_{1}}=\cdots=v_{i_{k}}=0\right\}$. Thus $\operatorname{codim} V^{0}=k_{0}:=\min \left\{k \mid \exists\left\{x_{i_{1}}, \ldots, x_{i_{k}}\right\}\right.$ satisfying $\left.\star\right\}$. Hence $(V, T)$ is equidimensional if and only if $k_{0}=\operatorname{dim} V / / T=n-r$.

Now $(V, T)$ is faithful implies that $\operatorname{span}_{\mathbb{Z}}\left\{\mu_{1}, \ldots, \mu_{n}\right\}=X^{*}(T) \cong \mathbb{Z}^{r}$. Reordering we may suppose that $\left\{\mu_{n-r+1}, \ldots, \mu_{n}\right\}$ is a $\mathbb{Q}$-basis for $\mathbb{Z}^{r} \otimes \mathbb{Q}$. Any monomial $m=x_{i_{1}}^{a_{1}} \cdots \cdots x_{i_{k}}^{a_{k}} \in \mathbb{C}[V]^{T}$ gives a relation: $a_{i_{1}} \mu_{i_{1}}+\cdots+a_{i_{k}} \mu_{i_{k}}=0$ in $\mathbb{Z}^{r}$. Since $\left\{\mu_{n-r+1}, \ldots, \mu_{n}\right\}$ is linearly independent, there can be no monomial $m \in \mathbb{C}[V]^{T}$ involving only $x_{n-r+1}, \ldots, x_{n}$. Equivalently, every monomial $m \in \mathbb{C}[V]^{T}$ involves at least one of $x_{1}, \ldots, x_{n-r}$. Therefore, $\left\{x_{1}, \ldots, x_{n-r}\right\}$ satisfies $\star$.

Now $(V, T)$ is equidimensional implies $k_{0}=n-r$ from which it follows that no proper subset of $\left\{x_{1}, \ldots, x_{n-r}\right\}$ satisfies property $\star$. This implies that for all $i$ with $1 \leq i \leq n-r$, there exists a $j$ such that $x_{i}$ divides $m_{j}$.

Fix $j \leq n-r$ and suppose that $w_{1}, \ldots, w_{q}$ are precisely those $m_{i}$ in which $x_{j}$ appears. If each of $w_{1}, \ldots, w_{q}$ is also divisible by at least one of $x_{1}, \ldots, \widehat{x_{j}}, \ldots, x_{n-r}$, then $\left\{x_{1}, \ldots, \widehat{x}_{j}, \ldots, x_{n-r}\right\}$ would satisfy property $\star$. Hence at least one of $w_{1}, \ldots, w_{q}$ is divisible by $x_{j}$ but not by any of $\left\{x_{1}, \ldots, \widehat{x_{j}}, \ldots, x_{n-r}\right\}$. Thus reordering the $m_{i}$ we may assume that $x_{j}$ divides $m_{i}$ for $1 \leq i, j \leq n-r$ if and only if $i=j$. Therefore we may write:

$$
\begin{array}{cc}
m_{1} & =x_{1}^{a_{1}} x_{n-r+1}^{a_{1, n-1}} \cdots \cdots x_{n}^{a_{1, n}} \\
m_{2} & =x_{2}^{a_{2}} x_{n-r+1}^{a_{2, n+1}} \cdots \cdots x_{n}^{a_{2, n}} \\
\vdots & \vdots \\
m_{n-r} & =x_{n-r}^{a_{n-r}} x_{n-r+1}^{a_{n-r, r+1}} \cdots \cdots x_{n}^{a_{n-r, n}}
\end{array}
$$

where $a_{1}, \ldots, a_{n-r} \geq 1$ and $a_{j, i} \geq 0$ for $1 \leq j \leq n-r$ and $n+r-1 \leq i \leq n$. 
This gives the following relations in $X^{*}(T) \cong \mathbb{Z}^{r}$ :

$$
\begin{array}{r}
a_{1} \cdot \mu_{1}+a_{1, n-r+1} \cdot \mu_{n-r+1}+\cdots+a_{1, n} \cdot \mu_{n}=0 \\
a_{2} \cdot \mu_{2}+a_{2, n-r+1} \cdot \mu_{n-r+1}+\cdots+a_{2, n} \cdot \mu_{n}=0 \\
\vdots \\
a_{n-r} \cdot \mu_{n-r}+a_{n-r, n-r+1} \cdot \mu_{n-r+1}+\cdots+a_{n-r, n} \cdot \mu_{n}=0
\end{array}
$$

Define $L_{j}:=\left\{n-r+1 \leq i \leq n \mid a_{j, i} \neq 0\right\} \sqcup\{j\}$ for $1 \leq j \leq n-r$. Then $i \in L_{j} \Longleftrightarrow x_{i}$ divides $m_{j}$ for $1 \leq i \leq n, 1 \leq j \leq n-r$.

Lemma 3. If $1 \leq u \neq v \leq n-r$, then $L_{u} \cap L_{v}=\varnothing$.

Proof. Assume $1 \leq u \neq v \leq n-r$ and $w \in L_{u} \cap L_{v}$. Clearly $n+r-1 \leq w \leq n$. Set $H:=\operatorname{span}_{\mathbb{Q}}\left\{\mu_{n-r+1}, \ldots, \widehat{\mu_{w}}, \ldots, \mu_{n}\right\}$, a hyperplane in $\mathbb{Z}^{r} \otimes \mathbb{Q}$. Then $m_{u}$ yields the relation

$$
a_{u} \cdot \mu_{u}+a_{u, w} \cdot \mu_{w} \equiv 0 \quad(\bmod H)
$$

where $a_{u} \geq 1$ and $a_{u, w} \geq 1$ since $w \in L_{u}$. Similarly, $m_{v}$ yields

$$
a_{v} \cdot \mu_{v}+a_{v, w} \cdot \mu_{w} \equiv 0 \quad(\bmod H)
$$

where $a_{v} \geq 1$ and $a_{v}, w \geq 1$ since $w \in L_{v}$.

Now $k_{0}=n-r$ implies that $\left\{x_{1}, \ldots, \widehat{x_{u}}, \ldots, \widehat{x_{v}}, \ldots, x_{n-r}, x_{w}\right\}$ does not satisfy property $\star$. Thus there is a monomial $m \in\left\{m_{1}, \ldots, m_{d}\right\}$ of the form $m=x_{u}^{a} \cdot x_{v}^{b} \cdot x_{n-r+1}^{b_{n}-r+1} \cdots \cdots x_{n}^{b_{n}}$ with $b_{w}=0, a \geq 0$, and $b \geq 0$. Hence $m$ yields the relation

$$
a \cdot \mu_{u}+b \cdot \mu_{v} \equiv 0 \quad(\bmod H)
$$

where $a \geq 0 ; b \geq 0$. From Equation (1) we see that $\mu_{u}$ and $\mu_{w}$ lie on opposite sides of the hyperplane $H$. Similarly Equation (2) shows that $\mu_{v}$ and $\mu_{w}$ lie on opposite sides of $H$. Hence $\mu_{u}$ and $\mu_{v}$ lie on the same side of $H$ and thus, in Equation (3), we must have $a=b=0$. Hence $m=x_{n-r+1}^{b_{n}-r+1} \cdots \cdots x_{n}^{b_{n}}$. Therefore $b_{n-r+1} \cdot \mu_{n-r+1}+\cdots+b_{n} \cdot \mu_{n}=0$. But $\left\{\mu_{n-r+1}, \ldots, \mu_{n}\right\}$ is linearly independent and thus $b_{n-r+1}=\cdots=b_{n}=0$. Thus $m=1 \in\left\{m_{1}, \ldots, m_{d}\right\}$, and this contradiction proves the lemma.

Set $M_{j}:=\operatorname{span}_{\mathbb{Z}}\left\{\mu_{i} \mid i \in L_{j}\right\}$. Since $m_{j}$ has weight zero, we have a relation among the elements of $\left\{\mu_{i} \mid i \in L_{j}\right\}$. Hence rank $M_{j} \leq \#\left(L_{j}\right)-1$. Since $(V, T)$ is faithful, we have $X^{*}(T)=\operatorname{span}_{\mathbb{Z}}\left\{\mu_{1}, \ldots, \mu_{n}\right\}=M_{1}+\cdots+M_{n-r}$.

Now

$$
\begin{aligned}
r & =\operatorname{rank} X^{*}(T)=\operatorname{rank}\left(M_{1}+\cdots+M_{n-r}\right) \leq \sum_{j=1}^{n-r} \operatorname{rank} M_{j} \\
& \leq \sum_{j=1}^{n-r}\left(\#\left(L_{j}\right)-1\right)=n-(n-r)=r .
\end{aligned}
$$

Hence rank $M_{j}=\#\left(L_{j}\right)-1$ for $j=1, \ldots, n-r$ and $X^{*}(T)=M_{1} \oplus \cdots \oplus M_{n-r}$. Define

$$
V_{j}:=\operatorname{span}_{\mathbb{C}}\left\{v_{i} \mid i \in L_{j}\right\} \text { and } T_{j}:=\bigcap_{\substack{x \in M_{i} \\ i \neq j}} \operatorname{ker} \chi .
$$

Then $T=T_{1} \times \cdots \times T_{n-r}$ and $X^{*}\left(T_{j}\right)=M_{j}$ by Lemma 1 . 
The monomial $m_{j}$ provides a linear relation over $\mathbb{N}$ among the weights of $V_{j}$. However, since $x_{i}$ divides $m_{j}$ for every $i \in L_{j}$, each weight of $V_{j}$ occurs in this relation and thus any $\operatorname{dim} V_{j}-1$ of them are linearly independent over $\mathbb{Q}$. Furthermore, $\left(V_{j}, T_{j}\right)$ is stable by Lemma 2 since $m_{j} \in \mathbb{C}\left[V_{j}\right]^{T_{j}}$. Since $T_{j_{1}}$ acts trivially on $V_{j_{2}}$, if $j_{1} \neq j_{2}$ and $(V, T)$ is faithful, $\left(V_{j}, T_{j}\right)$ must be faithful for all $j$. Thus $\operatorname{dim} V_{j} / / T_{j}=\operatorname{dim} V_{j}-\operatorname{dim} T_{j}=\operatorname{dim} V_{j}-\operatorname{rank} M_{j}=1$. From this it easily follows that $\left(V_{j}, T_{j}\right)$ is equidimensional and coregular and so cofree.

Remark 3. The above theorem combined with Lemma 2 gives a complete description of equidimensional torus representations in terms of their weights.

\section{The Popov conjecture}

Theorem 2 (The Popov conjecture for tori). Let $(V, T)$ be an equidimensional torus representation. Then $(V, T)$ is cofree.

Proof. By Lemma 2 we may assume that $(V, T)$ is faithful and stable. Hence Theorem 1 applies and $(V, T)$ decomposes as $(V, T)=\left(V_{1}, T_{1}\right) \oplus \cdots \oplus$ $\left(V_{n-r}, T_{n-r}\right)$ with each $\left(V_{j}, T_{j}\right)$ cofree. Therefore $(V, T)$ is coregular and equidimensional and so also cofree.

\section{REFERENCES}

1. O. M. Adamovich, Equidimensional representations of simple algebraic groups, Amer. Math. Soc. Transl. (2) 128 (1986), 25-29.

2. O. M. Adamovich and E. O. Golovina, Simple linear Lie groups having a free algebra of invariants, Selecta Math. Soviet 3 (1983/84), 183-220.

3. V. G. Kac, Some remarks on nilpotent orbits, J. Algebra 64 (1980), 190-213.

4. V. G. Kac, V. L. Popov, and E. B. Vinberg, Sur les groupes lineaires algébriques dont l'algèbre des invariants est libre, C. R. Acad. Sci. Paris Sér. I Math. 283 (1976), 865-878.

5. H. Kraft, Geometrische Methoden in der Invariantentheorie, Aspects of Math., Vieweg, Braunschweig, 1984.

6. P. Littelmann, Koreguläre und äquidimensionale Darstellungen, J. Algebra 123 (1989), 193-222.

7. D. Mumford and J. Fogarty, Geometric invariant theory (2nd enlarged ed.), Ergeb. Math./ Grenzgeb., vol. 34, Springer-Verlag, Berlin, 1982.

8. V. L. Popov, Representations with a free module of covariants, Funct. Anal. Appl. 10 (1976), 242-244.

9. G. W. Schwarz, Representations of simple Lie groups with regular rings of invariants, Invent. Math. 49 (1978), 167-191.

10. __ Representations of simple Lie groups with a free module of covariants, Invent. Math. 50 (1978), 1-12.

11. _ Lifting smooth homotopies of orbit spaces, Inst. Hautes Études Sci. Publ. Math. 51 (1980), 37-135.

12. T. A. Springer, Linear algebraic groups, PM 9, Birkhäuser Verlag, 1977.

13. D. L. Wehlau, Equidimensional representations and the Popov conjecture, Ph.D. Thesis, Brandeis University, 1989.

Department of Mathematics, University of Toronto, Toronto, Ontario, M5S 1A1 CANADA 